\title{
Risk factors for hemorrhage requiring embolization after percutaneous nephrolithotomy: a meta-analysis
}

\author{
Zhiqin $\mathrm{Li}^{1}$, Aiming $\mathrm{Wu}^{1}$, Jianjun Liu ${ }^{2}$, Shuitong Huang ${ }^{1}$, Guangming Chen ${ }^{1}$, Yonglu Wu ${ }^{1}$, Xianxi Chen ${ }^{1}$, \\ Guobin Tan ${ }^{1}$ \\ ${ }^{1}$ Department of Urology, Maoming People's Hospital, Maoming 525000, China; ${ }^{2}$ Department of Urology, Affiliated Hospital of Guangdong Medical \\ University, Zhanjiang 524000, China \\ Contributions: (I) Conception and design: G Tan; (II) Administrative support: G Tan; (III) Provision of study materials or patients: Z Li; (IV) \\ Collection and assembly of data: Z Li; (V) Data analysis and interpretation: Z Li, G Tan; (VI) Manuscript writing: All authors; (VII) Final approval of \\ manuscript: All authors. \\ Correspondence to: Guobin Tan. Department of Urology, Maoming People’s Hospital, Maoming 525000, China. Email: ilppdameinv@163.com.
}

\begin{abstract}
Background: The aim of this meta-analysis was to systematically review and identify the risk factors for severe hemorrhage after percutaneous nephrolithotomy (PCNL).

Methods: We searched the PubMed and EMBASE database for literature related to the risk factors of severe hemorrhage after PCNL requiring angiography and embolization through to September 2019. The necessary data for each eligible study were extracted by 2 independent reviewers. The Newcastle-Ottawa Scale (NOS) was used for assessing the methodological quality of the included studies. Statistical analyses were conducted using Comprehensive Meta-Analysis version 2 to identify whether there was a statistical association between risk factors and severe hemorrhage post-PCNL.

Results: The results of this meta-analysis showed that urinary tract infection (UTI) (OR =1.98, 95\% CI, 1.21-3.26, $\mathrm{P}=0.007$ ), diabetes mellitus ( $\mathrm{OR}=4.07$, 95\% CI, 1.83-9.06, $\mathrm{P}=0.001)$, staghorn stone $(\mathrm{OR}=3.49$, 95\% CI, 1.25-9.76, $\mathrm{P}=0.017)$, and multiple tracts ( $\mathrm{OR}=2.09,95 \% \mathrm{CI}, 1.33-3.28, \mathrm{P}=0.001)$ were independent risk factors for severe hemorrhage post-PCNL, while hypertension ( $\mathrm{OR}=1.18,95 \% \mathrm{CI}, 0.58-2.42, \mathrm{P}=0.65$ ) showed no significant statistical difference.

Conclusions: Urologists should focus on the above identified risk factors for severe hemorrhage postPCNL, including UTI, diabetes mellitus, staghorn stone, and multiple tracts. More high-quality studies with larger sample sizes are needed to validate these conclusions.
\end{abstract}

Keywords: Hemorrhage; percutaneous nephrolithotomy (PCNL); embolization; meta-analysis; risk factor

Submitted Dec 02, 2019. Accepted for publication Jan 06, 2020.

doi: $10.21037 /$ tau.2020.01.10

View this article at: http://dx.doi.org/10.21037/tau.2020.01.10

\section{Introduction}

Percutaneous nephrolithotomy (PCNL) was first described in 1976 by Fernstrom and Johansson and has seen widespread use with the technical development of endoscopic and auxiliary instruments (1). As a result of this progress and the overall increase in experience, PCNL is now recommended as first-line therapy for renal stones $>2 \mathrm{~cm}$ (2). While PCNL is considered a safe and effective procedure, it is still associated with the very serious complication of renal hemorrhage. The timing of this hemorrhage remains uncertain, as it can occur immediately after the procedure or only after a few days. Previous studies have found that embolization rates vary from $0.6 \%$ to $2.6 \%$, and transfusion rates vary from $1 \%$ to $11 \%$ after PCNL (3). As conservative treatment to hemostasis, including nephrostomy tube clamping, adequate hydration, hemostatic drugs, and blood transfusion, has been shown to be ineffective, transcatheter arterial embolization (TAE) has been recommended as a safe and effective method for severe 
hemorrhage after PCNL (4).

Although TAE has a preventive effect on hemorrhage for post-PCNL patients, early diagnosis and management are still necessary. Recently, more urologists have focused on patients with severe hemorrhage post-PCNL, with few studies having attempted to identify the association between risk factors and vascular complication post-PCNL. The relevant literature is able to indicate that urinary tract infection (UTI), hypertension, diabetes mellitus, number of tracts, and type of stone may be the important risk factors (5). However, few meta-analyses are available that have synthesized these data to provide convincing evidence for the association between risk factors and severe hemorrhage after PCNL.

Therefore, this meta-analysis was conducted to identify the risk factors for severe hemorrhage in post-PCNL patients with the aim of providing the confirming data to guide clinical practice.

\section{Methods}

\section{Literature search}

We searched the PubMed and EMBASE databases for literature concerning risk factors for severe hemorrhage after PCNL requiring angiography and embolization through to September 2019. The following search strategy was used for the search (in PubMed):

* \#1. Nephrolithotomy, Percutaneous

* \#2. Nephrolithotomies, Percutaneous

* \#3. Percutaneous Nephrolithotomies

* \#4. Percutaneous Nephrolithotomy

* \#5. PCNL

* \#6. PNL

* \#7. \#1 or \#2 or \#3 or \#4 or \#5 or \#6

* \#8. Embolism

* \#9. Embolization

* \#10. Transcatheter arterial embolization

* \#11. TAE

* \#12. \#8 or \#9 or \#10 or \#11

* \#13. Haemorrhage

* \#14. Hemorrhage

* \#15. Hematuria

* \#16. Bleeding

* \#17. \#13 or \#14 or \#15 or \#16

* \#18. \#7 and \#12 and \#17

Each obtained source was evaluated independently by two reviewers to ensure that the included studies were suitable.

\section{Study selection}

All studies meeting the following criteria were included: (I) the design type of the study was case-controlled; (II) the study focus was associated risk factors of severe hemorrhage required embolization after PCNL, with the odds ratios (ORs) and the corresponding 95\% confidence intervals (CIs), or the original data reported; (III) the language of study was English. All eligible studies found in the database search were independently evaluated by two reviewers according to the inclusion criteria. Non-case-control studies, systematic reviews, case reports, and duplicates were excluded. The relevant data for each eligible study, including first author's last name, year of publication, site of origin, source of controls, number of cases and controls, and associated risk factors were also independently extracted by the two reviewers. Any disagreements between the reviewers concerning inclusion/exclusion or data extraction of the articles were resolved by discussion or consultation with a third reviewer.

\section{Methodological quality assessment}

The data included first author, year of publication, type of study design, the number of cases and control, sample ages, and risk factors. During the data extraction, the authors were contacted if any information was missing or needed clarification. The Newcastle-Ottawa Scale (NOS) was used for assessing the methodological quality of the included studies, and is based on three categories: selection, comparability, and exposure or outcome. The NOS scores range from 0 to 9 stars, with 6 to 9 stars being considered a low risk of bias, 4 to 5 stars being considered a medium risk of bias, and 1 to 3 stars being considered a high risk of bias (6). The assessment results of methodological quality are summarized in Table 1 .

\section{Statistical analysis}

Statistical analyses were performed using Comprehensive Meta-Analysis version 2 (Biostat, Englewood, NJ, USA) to identify whether there was a statistical difference between risk factors and severe hemorrhage post-PCNL, to assess heterogeneity of the included studies, and to generate forest plots. For discontinuous variables, an OR with $95 \%$ CIs was calculated. Heterogeneity was calculated by using the $\chi^{2}$ and the $\mathrm{I}^{2}$ tests for included studies. A random-effects model was applied if significant heterogeneity was found among included studies $\left(\mathrm{P}<0.05\right.$ and $\left.\mathrm{I}^{2}>50 \%\right)$; otherwise, 
Table 1 Characteristics of the included studies

\begin{tabular}{|c|c|c|c|c|c|}
\hline Author/year & Study design & No. cases/control & Age (mean) & Risk factor & NOS score \\
\hline Arora et al. 2019 & Case control & $9 / 583$ & $44.45 \pm 11.95$ & (I), (II), (III), (IV), (V) & 8 \\
\hline Un et al. 2015 & Case control & $14 / 133$ & $42.5 \pm 9$ & (IV) & 8 \\
\hline Tan et al. 2013 & Case control & $10 / 982$ & Range $6-80$ years old & (IV), (V) & 8 \\
\hline Jinga et al. 2013 & Case control & $22 / 2,073$ & NR & (I), (IV), (V) & 8 \\
\hline El-Nahas et al. 2019 & Case control & $39 / 3,839$ & $50.7 \pm 12.6$ & (I), (IV) & 8 \\
\hline
\end{tabular}

Risk factors: (I) UTI; (II) hypertension; (III) diabetes mellitus; (IV) multiple tracts; (V) stone type. NR, no report.

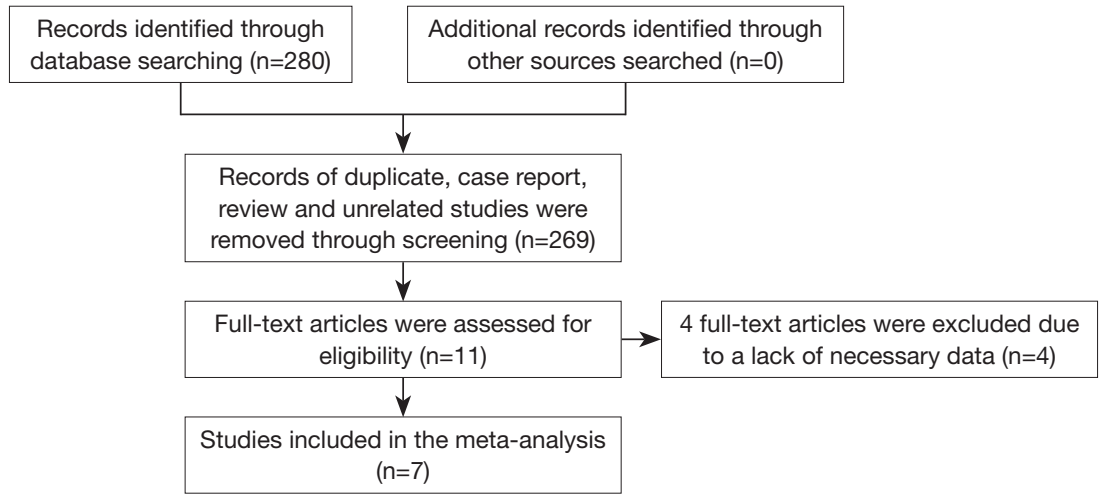

Figure 1 Flow diagram of the literature search.

a fixed-effects model was applied $\left(\mathrm{P}>0.05\right.$ and $\left.\mathrm{I}^{2}<50 \%\right)$. It was calculated from the original data, while the OR was not reported. Finally, publication bias was not assessed because the number of included studies was less than 10 .

\section{Results}

\section{Identification of eligible studies}

A total of 280 studies were identified in an initial search, among which 11 studies remained after removing duplicates, case reports, reviews, or unrelated studies by assessing the titles and abstracts. Finally, 7 studies were included, while the other 4 studies were excluded after further review because of a lack of relevant risk factors (Figure 1).

\section{Characteristics of included studies}

A total of 7 case-control studies published between 2007 and 2019 were included in the meta-analysis comprising 10,194 patients, 142 cases with severe hemorrhage, and 11,021 controls with non-severe hemorrhage. The sizes of sample ranged from 121 to 3,338 among included studies. While the mean ages of cases and controls were available in some studies (7), other studies (8) only indicated an age range or a mean age. All the studies were reported in English. Table 1 shows the characteristics of the included studies.

\section{Synthesis of the results}

After using the $\chi^{2}$ and the $\mathrm{I}^{2}$ test, no significant heterogeneity was identified in the four risk factors (UTI, hypertension, diabetes mellitus, and the number of tracts) except for stone type. Thus a fixed-effects model was generally applied, while a random-effects model was applied for stone type due to its statistically significant heterogeneity. The overall results are listed in Table 2. 
Table 2 Meta-analysis of the pooled risk factors for sever hemorrhage post-PCNL

\begin{tabular}{|c|c|c|c|c|c|c|c|c|c|}
\hline Risk factor & No. studies & No. cases & No. control & \multicolumn{2}{|c|}{ Heterogeneity test } & Effect model & OR (95\% Cl) & Z & $\mathrm{P}$ \\
\hline UTI & 4 & 78 & 6,068 & 0.31 & 15.74 & $\mathrm{~F}$ & $1.98(1.21,3.26)$ & 2.71 & $0.007^{\star \star}$ \\
\hline Diabetes mellitus & 2 & 41 & 672 & 0.59 & 0 & $\mathrm{~F}$ & $4.07(1.83,9.06)$ & 3.44 & $0.001^{\star \star}$ \\
\hline Hypertension & 2 & 41 & 682 & 0.55 & 0 & $\mathrm{~F}$ & $1.18(0.58,2.42)$ & 0.45 & 0.650 \\
\hline Staghorn stone & 3 & 71 & 6,884 & 0.04 & 67.06 & $\mathrm{R}$ & $3.49(1.25,9.76)$ & 2.38 & $0.017^{*}$ \\
\hline Multiple stone & 3 & 71 & 6,884 & 0.003 & 82.36 & $\mathrm{R}$ & $1.57(0.47,5.25)$ & 0.73 & 0.460 \\
\hline Single stone & 3 & 71 & 6,884 & 0.036 & 69.81 & $\mathrm{R}$ & $0.39(0.13,1.16)$ & -1.69 & 0.091 \\
\hline
\end{tabular}

*, P<0.05; **, P<0.01. Fixed effect model: F; Random effect model: R. PCNL, percutaneous nephrolithotomy; UTI, urinary tract infection.

\begin{tabular}{llllrrr} 
Study name & \multicolumn{5}{c}{ Statistics for each study } \\
\cline { 3 - 6 } & $\begin{array}{c}\text { Odds } \\
\text { ratio }\end{array}$ & $\begin{array}{c}\text { Lower } \\
\text { limit }\end{array}$ & $\begin{array}{c}\text { Upper } \\
\text { limit }\end{array}$ & Z-Value & p-Value \\
Du 2019 & 2.571 & 1.118 & 5.912 & 2.224 & 0.026 \\
Arora 2019 & 0.904 & 0.186 & 4.402 & -0.125 & 0.901 \\
El Tayeb 2014 & 1.057 & 0.375 & 2.978 & 0.106 & 0.916 \\
Jinga 2013 & 2.975 & 1.238 & 7.148 & 2.438 & 0.015 \\
& 1.984 & 1.209 & 3.256 & 2.712 & 0.007
\end{tabular}

\section{Odds ratio and $95 \% \mathrm{Cl}$}

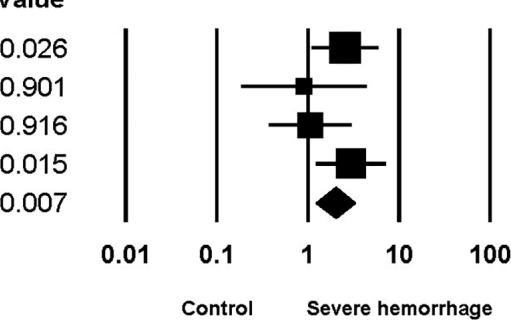

Figure 2 Forest plot: effect of UTI on severe hemorrhage post-PCNL. UTI, urinary tract infection; PCNL, percutaneous nephrolithotomy.

\section{Risk factors for severe hemorrbage post-PCNL}

\section{UTI}

Four studies $(2,4,7,9)$ reported the association between UTI and severe hemorrhage post-PCNL, among which low heterogeneity was found $\left(\mathrm{I}^{2}=15.74 \%, \mathrm{P}=0.31\right)$, along with significant difference $(\mathrm{OR}=1.94,95 \%$ CI, 1.209-3.256, $\mathrm{P}=0.007$ ) (Figure 2).

\section{Hypertension}

Two studies $(2,7)$ reported the association between hypertension and severe hemorrhage post-PCNL, among which low heterogeneity was found $\left(\mathrm{I}^{2}=0 \%, \mathrm{P}=0.55\right)$, but no significant difference $(\mathrm{OR}=1.180,95 \% \mathrm{CI}, 0.576-2.418$, $\mathrm{P}=0.651)$ (Figure 3).

\section{Diabetes mellitus}

Two studies $(2,7)$ reported the association between diabetes mellitus and severe hemorrhage post-PCNL, among which low heterogeneity was found $\left(\mathrm{I}^{2}=0 \%, \mathrm{P}=0.59\right)$, along with significant difference $(\mathrm{OR}=4.074,95 \% \mathrm{CI}, 1.832-9.063$, $\mathrm{P}=0.001$ ) (Figure 4).

\section{Multiple tracts}

Seven studies (2,4,7-11) reported the relationship between multiple tracts and the risk of severe hemorrhage postPCNL, among of which low heterogeneity was found $\left(\mathrm{I}^{2}=44.12 \%, \mathrm{P}=0.1\right)$, along with significant difference in the calculation of the study effect size $(\mathrm{OR}=2.089,95 \% \mathrm{CI}$, 1.331-3.278, $\mathrm{P}=0.001$ ) (Figure 5).

\section{Stone type}

Three studies (8-10) reported the association between staghorn stone and severe hemorrhage post-PCNL, with high heterogeneity $\left(\mathrm{I}^{2}=67.06 \%, \mathrm{P}=0.04\right)$ and significant statistical difference $(\mathrm{OR}=3.487,95 \%$ CI, 1.246-9.760, $\mathrm{P}=0.017$ ) (Figure 6) being found among the included studies; apart from this, no significant statistical difference was found between multiple stone and non-multiple stone (OR 


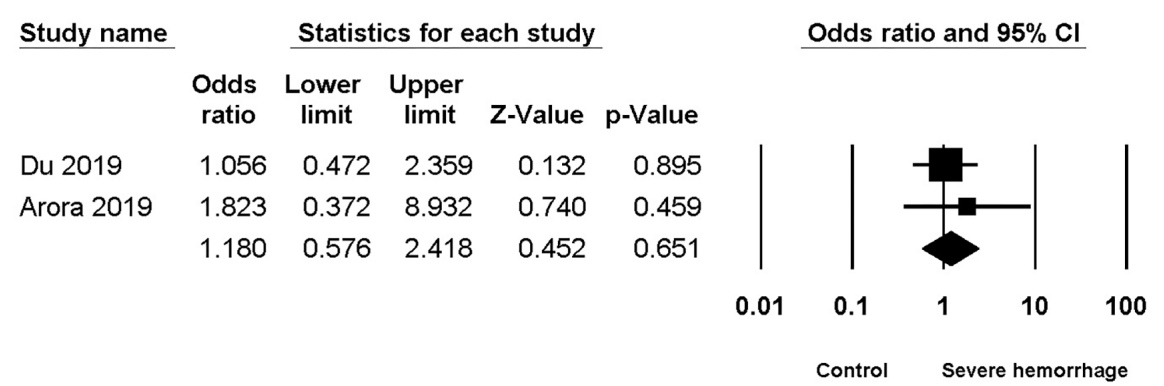

Figure 3 Forest plot: effect of hypertension for severe hemorrhage post-PCNL. PCNL, percutaneous nephrolithotomy.

\begin{tabular}{|c|c|c|c|c|c|c|c|c|c|}
\hline Study name & & Statisti & tics for ea & ach study & & & Odds 1 & o and $95 \% \mathrm{Cl}$ & \\
\hline & $\begin{array}{l}\text { Odds } \\
\text { ratio }\end{array}$ & $\begin{array}{c}\text { Lower } \\
\text { limit }\end{array}$ & $\begin{array}{l}\text { Upper } \\
\text { limit }\end{array}$ & Z-Value & p-Value & & & & \\
\hline Du 2019 & 4.740 & 1.793 & 12.530 & 3.137 & 0.002 & & & & \\
\hline Arora 2019 & 2.970 & 0.729 & 12.106 & 1.519 & 0.129 & & & & \\
\hline & 4.074 & 1.832 & 9.063 & 3.444 & 0.001 & & & & \\
\hline & & & & & & 0.01 & 0.1 & 10 & 100 \\
\hline
\end{tabular}

Figure 4 Forest plot: effect of diabetes mellitus for severe hemorrhage post-PCNL. PCNL, percutaneous nephrolithotomy.

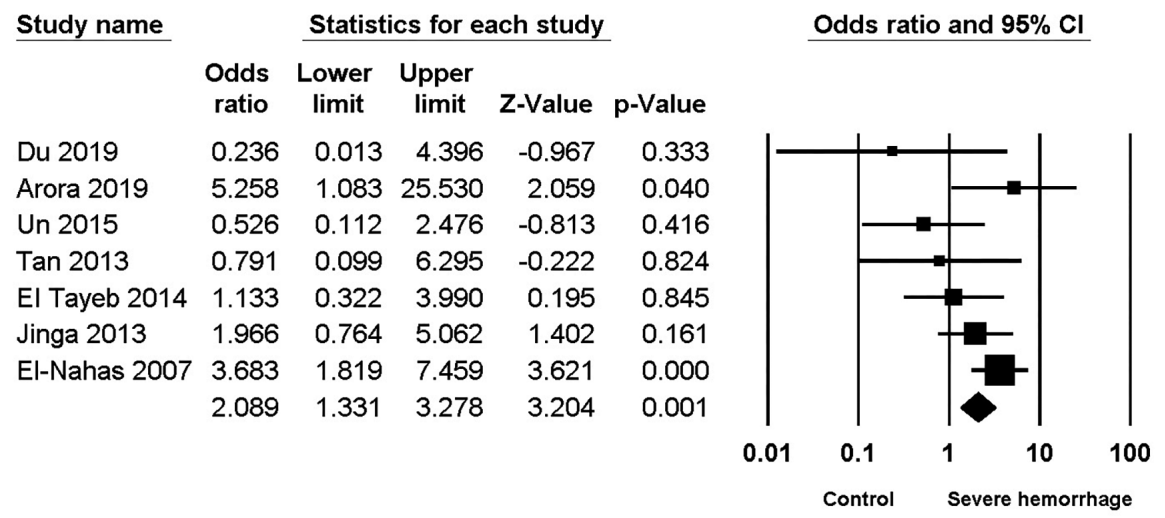

Figure 5 Forest plot: effect of multiple tracts for severe hemorrhage post-PCNL. PCNL, percutaneous nephrolithotomy.

$=1.569,95 \%$ CI, 0.469-5.247, $\mathrm{P}=0.464$ ) (Figure 7), with the same being the case for solitary stone $v s$. non-solitary stone (OR $=0.39,95 \%$ CI, 0.13-1.16, $\mathrm{P}=0.091$ ) (Figure 8).

\section{Discussion}

PCNL is an effective and safe operation for renal stones, and severe hemorrhage is a rarely associated vascular complication $(12,13)$. Angiography and embolization have been established for severe, persistent, or intermittent hemorrhage post-PCNL that cannot be stopped by conservative treatment $(14,15)$. In our meta-analysis, risk factors for severe hemorrhage post-PCNL requiring embolization included UTI, hypertension, diabetes mellitus, number of tracts, and stone type. Significant differences were found in UTI, diabetes mellitus, number of tracts, and 


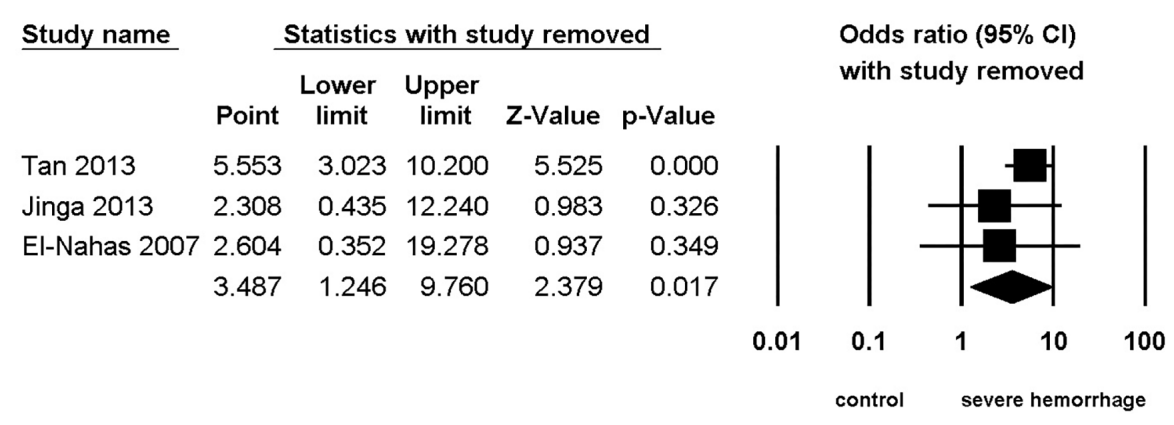

Figure 6 Forest plot: effect of staghorn stone for severe hemorrhage post-PCNL. PCNL, percutaneous nephrolithotomy.

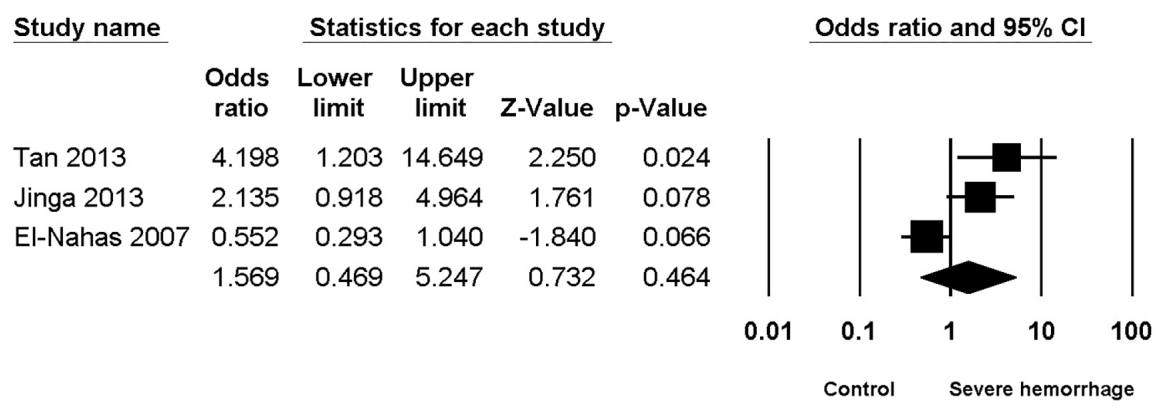

Figure 7 Forest plot: effect of multiple stone for severe hemorrhage post-PCNL. PCNL, percutaneous nephrolithotomy.

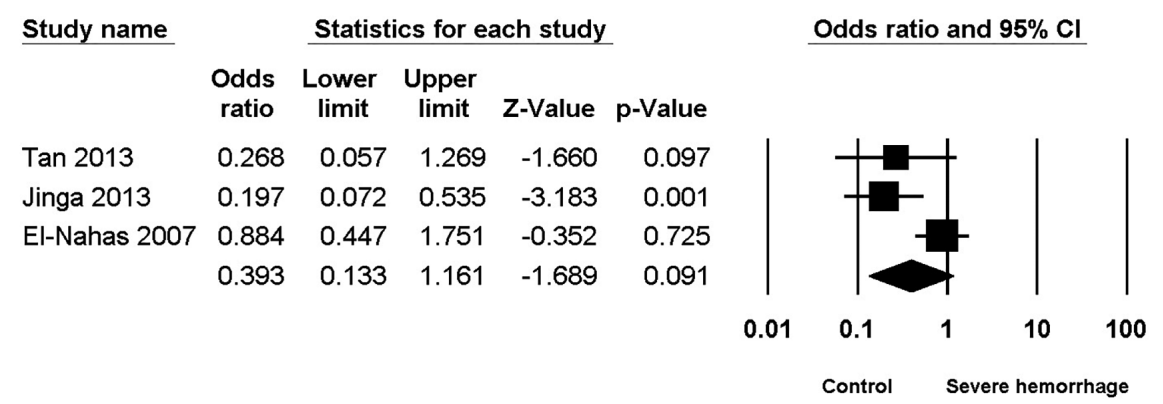

Figure 8 Forest plot: effect of solitary stone for severe hemorrhage post-PCNL. PCNL, percutaneous nephrolithotomy.

stone type, but not hypertension.

The risk of UTI in severe hemorrhage patients requiring embolization was 1.98 times higher than that in the control group (OR $=1.98,95 \% \mathrm{CI}, 1.21-3.26, \mathrm{P}=0.007)$, and was defined as the hyperemic nature of an inflamed urothelium or a distorted anatomy secondary to edema. However, among the studies included in this meta-analysis, no studies $(2,4,7,9)$ indicated a relation between UTI and severe hemorrhage post-PCNL, with some authors speculating this negative finding to possibly be a result of a small sample size. Du et al. (7) reported a higher risk of severe hemorrhage post-PCNL in patients with diabetes mellitus, while the other study (2) showed a negative result. Our result showed the pooled data $(\mathrm{OR}=4.07,95 \%$ CI, 1.83-9.06, $\mathrm{P}=0.001$ ) of our result was based on two studies, in which $36.59 \%(15 / 41)$ of patients in the severe hemorrhage post-PCNL group had a more frequent history of diabetes mellitus compared with $13.99 \%$ (94/672) in the control group. Previous studies showed that the mechanism of diabetes mellitus influencing the occurrence of severe 
hemorrhage post-PCNL can be explained by it affecting the whole vascular system and causing microangiopathies, which are highly vulnerable to bleeding after the initial trauma of tract formation (16-18).

Some studies $(2,9,10)$ indicate that the presence of staghorn stones is a risk factor for severe hemorrhage postPCNL, because during operation for such complex stones multiple tracts and excessive manipulation were needed. Our study also found the risk of severe hemorrhage postPCNL with staghorn stone to be increased $(\mathrm{OR}=3.49$, 95\% CI, 1.25-9.76, $\mathrm{P}=0.017)$. In addition, the risk of severe hemorrhage post-PCNL with multiple stone or solitary stone was also calculated, and the results indicated the risk of severe hemorrhage post-PCNL for either multiple stone $(\mathrm{OR}=1.57,95 \% \mathrm{CI}, 0.47-5.25, \mathrm{P}=0.46$ ) or solitary stone $(\mathrm{OR}=0.39,95 \% \mathrm{CI}, 0.13-1.16, \mathrm{P}=0.091) \mathrm{did}$ not increase. As for these results, we reasoned that larger calculi, especially staghorn calculi, often require prolonged operation time and multi-channel establishment, which significantly increases the damage to renal parenchyma, thereby increasing the risk of intraoperative and postoperative bleeding.

Finally, severe hemorrhage post-PCNL patients with multiple tracts had a 2.09 times higher risk of severe hemorrhage than patients with single tract $(\mathrm{OR}=2.09$, 95\% CI, 1.33-3.28, $\mathrm{P}=0.001)$. In clinical practice, we establish multiple tracts for a higher rate of stone cleaning, which leads to a higher risk of severe hemorrhage. The phenomenon of multiple tracts for higher stone cleaning combined with the higher risk of severe hemorrhage is confusing to nearly every urology surgeon.

The conclusion of this meta-analysis was reached based on seven studies, and offers more significant and statistical advice for the majority of first-line urologists. However, more research should be conducted to validate our conclusions.

This study has some limitations. First, some studies did not provide raw data, and errors might have occurred during data conversion. Secondly, due to the limited number of studies, we did not conduct sensitivity and funnel plot analyses. Third, only English language literature was retrieved. Finally, an inherent limitation is the small number of studies, so further research is needed to complete the meta-analysis.

The results of this meta-analysis showed that UTI, hypertension, diabetes mellitus, stone type, and multiple tracts were risk factors for severe hemorrhage post-PCNL. Urologists can focus on the association between identified risk factors and severe hemorrhage post-PCNL so that patients can be treated in time. The above conclusions may be biased because of the limited quality and quantity of the included studies. The correlation between the above risk factors and severe hemorrhage post-PCNL needs further study.

\section{Acknowledgments}

Funding: This study was funded by the Guangdong Medical Science and Technology Research Fund (No. 2017101819316701).

\section{Footnote}

Conflicts of Interest: All authors have completed the ICMJE uniform disclosure form (available at http://dx.doi. org/10.21037/tau.2020.01.10). The authors have no conflicts of interest to declare.

Ethical Statement: The authors are accountable for all aspects of the work in ensuring that questions related to the accuracy or integrity of any part of the work are appropriately investigated and resolved.

Open Access Statement: This is an Open Access article distributed in accordance with the Creative Commons Attribution-NonCommercial-NoDerivs 4.0 International License (CC BY-NC-ND 4.0), which permits the noncommercial replication and distribution of the article with the strict proviso that no changes or edits are made and the original work is properly cited (including links to both the formal publication through the relevant DOI and the license). See: https://creativecommons.org/licenses/by-ncnd/4.0/.

\section{References}

1. Fernström I, Johansson B. Percutaneous pyelolithotomy. A new extraction technique. Scand J Urol Nephrol 1976;10:257-9.

2. Arora AM, Pawar PW, Tamhankar AS, et al. Predictors for severe hemorrhage requiring angioembolization post percutaneous nephrolithotomy: A single-center experience over 3 years. Urol Ann 2019;11:180-6.

3. Seitz C, Desai M, Häcker A, et al. Incidence, prevention, and management of complications following percutaneous nephrolitholapaxy. Eur Urol 
2012;61:146-58.

4. El Tayeb MM, Knoedler JJ, Krambeck AE, et al. Vascular complications after percutaneous nephrolithotomy: 10 years of experience. Urology 2015;85:777-81.

5. Keoghane SR, Cetti RJ, Rogers AE, et al. Blood transfusion, embolisation and nephrectomy after percutaneous nephrolithotomy (PCNL). BJU Int2013;111:628-32.

6. Stang A. Critical evaluation of the Newcastle-Ottawa scale for the assessment of the quality of nonrandomized studies in meta-analyses.Eur J Epidemiol 2010;25:603-5.

7. Du N, Ma JQ, Luo JJ, et al. The Efficacy and Safety of Transcatheter Arterial Embolization to Treat Renal Hemorrhage after Percutaneous Nephrolithotomy. Biomed Res Int 2019;2019:6265183.

8. El-Nahas AR, Shokeir AA, El-Assmy AM, et al. Postpercutaneous nephrolithotomy extensive hemorrhage: a study of risk factors. J Urol 2007;177:576-9.

9. Jinga V, Dorobat B, Youssef S, et al. Transarterial embolization of renal vascular lesions after percutaneous nephrolithotomy. Chirurgia 2013;108:521-9.

10. Tan J, Chen B, He L, et al. Renal access through the inferior calyx is associated with higher risk of severe bleeding after percutaneous nephrolithotomy. Arch Med Sci 2015;11:340-5.

11. Un S, Cakir V, Kara C, et al. Risk factors for hemorrhage requiring embolization after percutaneous

Cite this article as: $\mathrm{Li} \mathrm{Z,} \mathrm{Wu} \mathrm{A,} \mathrm{Liu} \mathrm{J,} \mathrm{Huang} \mathrm{S,} \mathrm{Chen} \mathrm{G,}$ Wu Y, Chen X, Tan G. Risk factors for hemorrhage requiring embolization after percutaneous nephrolithotomy: a metaanalysis. Transl Androl Urol 2020;9(2):210-217. doi: 10.21037/ tau.2020.01.10 nephrolithotomy. Can Urol Assoc J 2015;9:E594-8.

12. Cai C, Liu Y, Zhong W, et al. The Clinical Application of New Generation Super-Mini Percutaneous Nephrolithotomy in the Treatment of $>/=20 \mathrm{~mm}$ Renal Stones. J Endourol 2019;33:634-8.

13. Poyraz N, Balasar M, Gokmen IE, et al. Clinical efficacy and safety of transcatheter embolization for vascular complications after percutaneous nephrolithotomy. Wideochir Inne Tech Maloinwazyjne 2017;12:403-8.

14. Aghamir SM, Elmimehr R, Modaresi SS, et al. Comparing Bleeding Complications of Double and Single Access Totally Tubeless PCNL: Is It Safe to Obtain More Accesses? Urol Int 2016;96:73-6.

15. Li L, Zhang Y, Chen Y, et al. A multicentre retrospective study of transcatheter angiographic embolization in the treatment of delayed haemorrhage after percutaneous nephrolithotomy. Eur Radiol 2015;25:1140-7.

16. Tefekli A, Kurtoglu H, Tepeler K, et al. Does the metabolic syndrome or its components affect the outcome of percutaneous nephrolithotomy? J Endourol 2008;22:35-40.

17. Akman T, Binbay M, Sari E, et al. Factors affecting bleeding during percutaneous nephrolithotomy: single surgeon experience. J Endourol 2011;25:327-33.

18. Kurtulus F, Fazlioglu A, Tandogdu Z, et al. Analysis of factors related with bleeding in percutaneous nephrolithotomy using balloon dilatation. Can J Urol 2010;17:5483-9. 